\title{
Interview with Professor Mark Wilcox
}

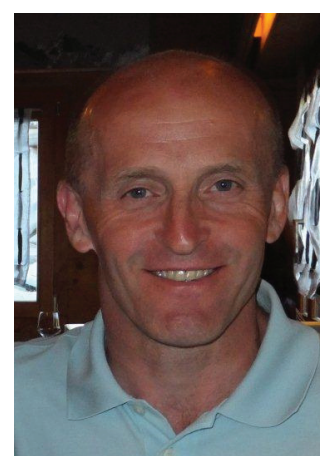

Mark Wilcox* speaks to Georgia Patey, Commissioning Editor: Professor Mark Wilcox is a Consultant Microbiologist and Head of Microbiology at the Leeds Teaching Hospitals (Leeds, UK), the Professor of Medical Microbiology at the University of Leeds (Leeds, UK), and is the Lead on Clostridium difficile and the Head of the UK C. difficile Reference Laboratory for Public Health England (PHE). He was the Director of Infection Prevention (4 years), Infection Control Doctor (8 years) and Clinical Director of Pathology (6 years) at the Leeds Teaching Hospitals. He is Chair of PHE's Rapid Review Panel (reviews utility of infection prevention and control products for National Health Service), Deputy Chair of the UK Department of Health's Antimicrobial Resistance and Healthcare Associated Infection Committee and a member of PHE's HCAI/AR Programme Board. He is a member of UK/European/US working groups on C. difficile infection. He has provided clinical advice as part of the FDA/EMA submissions for the approval of multiple novel antimicrobial agents. He heads a healthcare-associated infection research team at University of Leeds, comprising approximately 30 doctors, scientists and nurses; projects include multiple aspects of $C$. difficile infection, diagnostics, antimicrobial resistance and the clinical development of new antimicrobial agents. He has authored more than 400 publications, and is the coeditor of Antimicrobial Chemotherapy (5th/6th/7th Editions, 15 December 2007).

First draft submitted: 29 April 2016; Accepted for publication: 22 June 2016;

Published online: 5 August 2016

Q Could you briefly summarize your career to date?

I am currently the Head of Microbiology, teaching in hospital trusts in Leeds (UK). I am also a Professor of Medical Microbiology at the University of Leeds and I run a large research team focusing on healthcare-associated infections, in particular Clostridium diffcile infection (CDI). The third part of my job is working for Public Health England. I am the lead on $C$. diffcicile for the control, monitoring and management in England. Thus, my career to date has really brought me to this three-way split role that I have in the local National Health Service, research academic role and the national policy role, which has over the years increasingly focused on CDI and how the epidemiology of C. difficile has changed.

Q Can you tell me a bit more about your role as the Lead on C. difficile for Public Health England? What are you working on at the moment?

My role as the $C$. difficile lead involves several different aspects in $C$. difficile control. I run the $C$. difficile typing network, which is the way we map the types of $C$. difficile that are causing infections in the UK. We look to see what is controlling the strain that is

*Leeds Teaching Hospitals NHS Trust, Department of Microbiology, Old Medical School, LGI, Leeds, LS1 3EX; and University of Leeds, Leeds, LS2 9JT, UK; mark.wilcox@leedsth.nhs.uk

\section{KEYWORDS \\ $-\mathrm{CDI} \bullet$ C. difficile $\bullet$ comorbidities \\ - recurrence}

Future $\because \%$ 
particularly associated with poor outcomes and we are constantly monitoring to see if there are any new emergent strains. That role has actually spilled into monitoring strains throughout Europe and other parts of the world. Different countries and different healthcare systems are at varying stages of the $C$. difficile story. Some are along the way up to their peak; some are at the peak; and some have come down to the other side of that peak. Thus, it is important to be able to help those in the different scenarios and hopefully give them some advice based on what we have gone through in the UK about how best to control $C$. difficile.

The other parts of my role are to lead on producing the national guidelines on how to diagnose $C$. difficile. In the UK, we probably have better standardization for $C$. difficile diagnosis than any other country in the world. This is through research that was carried out in four different National Health Service hospitals, which led to the national guidance in 2012. This has since led to other major publications that have influenced European and probably North American diagnosis guidelines.

Lastly, I lead on the $C$. difficile treatment guidelines in the UK, which were last updated in 2013. I think with the imminent arrival of other therapeutic options those guidelines will need to be revisited. This will specifically give better information on how to use each of the treatment options for the different patient groups. It is worth noting that in the past a very commonly prescribed antibiotic for $C$. difficile was metronidazole and the guidelines reflect that. However, there is increasing evidence that metronidazole is an inferior option to vancomycin or fidaxomicin. Therefore, I would predict that in future guidelines there will be a firmer recommendation about when to use and when not to use that metronidazole treatment.

Q Why is CDI such a serious healthcare issue? What is the clinical \& economic impact of CDI? CDI is an important infection. It is one of the most common healthcare-associated infections worldwide. Indeed, in some countries it is the most commonly reported healthcare-associated infection. It is associated with considerable morbidity, mortality and excess cost. The most recent UK data available are that the 30-day mortality after diagnosis of CDI is approximately $16 \%$ and approximately half of those patients appear to die of CDI directly. That is a very high burden
- there are a few diseases that carry such high mortality rates. Of course, this high mortality partly reflects the fact that CDI tends to occur in the frail elderly who often have multiple comorbidities, so this may be an issue of 'the straw that breaks the camel's back.' Nevertheless, regardless of the baseline status of the individuals that are infected, the evidence suggests that there is significant excess mortality, which, in theory, could be prevented if either we stopped CDI happening in the first place or we had optimized treatment options that could improve outcomes.

In terms of cost, there are multiple studies that have been performed. Obviously, the cost tends to vary according to healthcare systems. The main determinant of cost is the increased length of hospital stay. In Europe, that increased stay is typically between 1 and 2 weeks over the baseline length of stay for whatever condition brought them into hospital. Thus, the average cost of a primary CDI episode, if you add up the increased length of stay, drug cost, test cost, etc., is approximately $£ 6,000-8,000$ to $£ 10,000$ pounds per episode, with a similar cost for recurrent episodes as well.

\section{Q How does CDI in the UK compare with the rest of the world?}

The epidemiology of CDI varies quite markedly from country to country, and if you think of that epidemiology as being a journey, many countries are seeing high current rates of CDI and indeed rates are increasing; European countries, such as Spain and Germany, would be good examples of that. The USA currently has very high rates that have been increasing in the last few years. In the UK, by contrast, CDI remains to be an issue but the rates have, for a considerable number of years now, been declining. We had peak rates in 2007/2008 and, on the back of a very major public health campaign, we have seen a $70-80 \%$ reduction in CDI rates and associated mortality. However, over the last 2-3 years, the rates have plateaued and the most recent data available to us suggest a small increase in CDI rates in the UK of approximately 6\%; that was the year 2014/2015 when compared with the previous 12 months.

\section{Q You mentioned that the elderly are at risk of CDI. Why is this? Are there any other population groups that are more at risk of CDI than others? \\ Yes, primarily the elderly are at risk of CDI, especially the elderly with multiple comorbidities.}


This is because multiple comorbidities make it more likely that you will be hospitalized and you are more likely to come into contact with CDI when you are hospitalized. If you are hospitalized, you are also more likely to be treated with antibiotics, but antibiotics are a major risk factor for CDI. There are some other key groups that do not fit neatly into the elderly, frail, comorbidity group; those are the patients who are undergoing epidemiological malignancies (typically bone marrow transplants or chemotherapy), as well as the patients with chronic renal failure who are undergoing dialysis. Sometimes we see outbreaks in other settings, for example, in surgical units with patients undergoing gut surgery. All patients who are admitted acutely to hospital tend to be at an increased risk of CDI. In addition, other key groups at risk are those with inflammatory bowel disease (IBD), Crohn's disease and ulcerative colitis. These are very difficult groups to study because of their baseline symptoms of diarrhea and/or colitis, and working out whether $C$. diffcicile adds to these baseline symptoms is very difficult. However, the data do suggest that patients with IBD who do test positive for $C$. difficile tend to have worse symptoms or outcomes.

\section{Q What can you tell me about your current research?}

I have recently been involved in a questionnairebased survey that was carried out across a large range of different medical professions, working in different countries, where we were trying to determine the level of understanding about CDI and IBD. What the research showed was that while there was quite a high level of agreement about some aspects about CDI, particularly about its potential to cause IBD, there were key areas of uncertainty among professionals. Importantly, this included those professionals who treat and manage IBD patients on a day-today basis. Levels of uncertainty were regarding how to make an accurate diagnosis of CDI in patients who already had symptoms of diarrhea and colitis. Moreover, the area of most controversy was about the optimal treatment of CDI in IBD patients.

Q How, in your opinion, do you think we can improve the management of $C D I$ in patients with IBD?

I think the survey study results clearly demonstrate that there is a need for more information to clarify this issue. We need a more careful study of IBD patients who do test positive for CDI to try and determine what additional impact this is causing. Ideally, we would particularly benefit from a study where patients are randomized to be given treatment or to not be given treatment. That is ethically difficult to do in some patients who have particularly concerning symptoms; however, there are ways this may be possible, whether their symptoms are severe or not. Then, by carefully following individuals according to whether they are randomized to receive the treatment or not, one could work out whether the initiation of treatment altered the outcome, shortened the time to resolve symptoms, shortened hospitalization and perhaps reduced the need for additional anti-inflammatory treatment, steroids, biologics, etc. Thus, there is a considerable scope to understand how we can optimize treatment. However, it is such a difficult area, and so people have tended to shy away from doing these types of studies, but I think it is getting harder and harder to avoid doing them to get the answer we need.

\section{Q Recurrence is a major difficulty in treating CDI. How do you think we can reduce, or even prevent, recurrence?}

Recurrence is one of the major hallmarks for hospitalized CDI; approximately a quarter of patients will experience a recurrent episode. If you have had one recurrence, you are more likely to get a second recurrence ( $40 \%$ chance), and if you have a second recurrence you have up to a $60 \%$ chance of getting a third recurrence. Therefore, there is a high baseline risk of recurrence that escalates if you are unlucky enough to experience a recurrence. Particularly, as we have already discussed, if there is a patient with multiple comorbidities with little capacity to cope with CDI, these recurrences can have very serious consequences.

What we do know is that of the three main treatment options currently available for $C$. difficile (metronidazole, vancomycin and fidaxomicin), fidaxomicin reduces the risk of recurrence by approximately $50 \%$. Therefore, with a $25 \%$ risk of occurrence, one is down to an approximately $12-13 \%$ risk of recurrence. The key to reducing recurrence is ensuring patients who are most likely to benefit from the reduced recurrence risk and are treated with fidaxomicin. We can identify patients with an increased risk of recurrence by using a short list of risk factors: 
patients who have already had a recurrence, those who have multiple comorbidities, the elderly and individuals who are on concomitant antibiotics (antibiotics other than those used to treat CDI). Similarly, those who have severe CDI episodes and/or those who are immunocompromised tend to have more recurrences. Consequently, these are the groups where we will be focusing on using the best treatment option currently available to reduce the risk of recurrence.

\section{Q Where do you see this field of research going in the next 10 years? Are there any particular areas where further research is needed?}

There are a number of treatment options currently being investigated, which range from antibiotics to antibodies. There are preventative options that are also undergoing clinical trials, including the vaccines, antibiotics, binding resins and, possibly, substances that could destroy antibiotics in the gut and, therefore, prevent their effects on the gut bacteria that are prone to $C$. difficile colonization. As we get a better choice of treatment options, we will need to get better at deciding which patients should receive which treatment. It has not really been a huge issue up until now because we have had so few treatment options; however, we should be distinguishing which patients should receive each of the three treatment options. That problem will only become greater as more treatment options become available.

The other issue that we discussed investigating was how best to manage CDI in key patient groups, in particular IBD patient groups where there is a lot we need to learn. I think we could learn more about trying to monitor the effectiveness of treatments. If we get groups who might be at risk of poor outcomes, such as severe disease, recurrence or indeed death, we could perhaps give combination treatments to reduce the risk of these poor outcomes.

Lastly, we still need to understand more about preventing CDI. We already know quite a lot about how $C$. difficile is transmitted in a hospital setting, but we have more to learn about how it is transmitted within and between individuals in a community setting. Of course, this is relevant because patients who are constantly being admitted into hospital may be bringing $C$. difficile into hospital with them. This provides constant replenishment of $C$. difficile strain types in the hospital setting, all of which do not necessarily behave in the same way. Therefore, we need to understand more about that whole process.

\section{Disclaimer}

The opinions expressed in this interview are those of the interviewee and do not necessarily reflect the views of Future Medicine Ltd.

\section{Financial \& competing interests disclosure}

Abbott, Actelion, Alere, Astellas, Biomerieux, Cerexa, Cubist, Da Volterra, European Tissue Symposium, Merck, Sanofi-Pasteur, Summit, The Medicines Company and Qiagen have funded research by the author in the past 2 years. The author has received consultancies and/or lecture honoraria in the past 2 years from Actelion, Alere, Astellas, Astra-Zeneca, Basilea, Bayer, Cubist, Durata, European Tissue Symposium, J\&J, Merck, Nabriva, Novacta, Novartis, Optimer, Pfizer, Roche, Sanofi-Pasteur and Seres. The author is part of the Speaker's Bureau for Pfizer. The author has no other relevant affiliations or financial involvement with any organization or entity with a financial interest in or financial conflict with the subject matter or materials discussed in the manuscript apart from those disclosed.

No writing assistance was utilized in the production of this manuscript. 